\title{
Universal Compressive Characterization of Quantum Dynamics
}

\author{
Yosep Kim $\odot,{ }^{1}$ Yong Siah Teo ${ }^{2,},{ }^{*}$ Daekun Ahn, ${ }^{2}$ Dong-Gil Im, ${ }^{1}$ Young-Wook Cho $\odot,{ }^{3}$ Gerd Leuchs $\odot,{ }^{4,5}$ \\ Luis L. Sánchez-Soto $\oplus^{4,6}$ Hyunseok Jeong, ${ }^{2, \dagger}$ and Yoon-Ho Kim $\oplus^{1, \ddagger}$ \\ ${ }^{1}$ Department of Physics, Pohang University of Science and Technology (POSTECH), 37673 Pohang, Korea \\ ${ }^{2}$ Department of Physics and Astronomy, Seoul National University, 08826 Seoul, Korea \\ ${ }^{3}$ Center for Quantum Information, Korea Institute of Science and Technology (KIST), 02792 Seoul, Korea \\ ${ }^{4}$ Max-Planck-Institut für die Physik des Lichts, Staudtstraße 2, 91058 Erlangen, Germany \\ ${ }^{5}$ Institute of Applied Physics, Russian Academy of Sciences, 603950 Nizhny Novgorod, Russia \\ ${ }^{6}$ Departamento de Óptica, Facultad de Física, Universidad Complutense, 28040 Madrid, Spain
}

(Received 11 January 2020; accepted 27 April 2020; published 27 May 2020)

\begin{abstract}
Recent quantum technologies utilize complex multidimensional processes that govern the dynamics of quantum systems. We develop an adaptive diagonal-element-probing compression technique that feasibly characterizes any unknown quantum processes using much fewer measurements compared to conventional methods. This technique utilizes compressive projective measurements that are generalizable to an arbitrary number of subsystems. Both numerical analysis and experimental results with unitary gates demonstrate low measurement costs, of order $O\left(d^{2}\right)$ for $d$-dimensional systems, and robustness against statistical noise. Our work potentially paves the way for a reliable and highly compressive characterization of general quantum devices.
\end{abstract}

DOI: $10.1103 /$ PhysRevLett.124.210401

Introduction.-Quantum processes are nature's directives that guide the evolution of all physical systems in the quantum realm. Such processes ubiquitously occur in untamed open-system dynamics under interactions with the environment as, for example, depolarizing [1], dephasing [2], and photon-loss [3] channels. They also exist as universal gates to carry out quantum computation. Notably, quantum processors [4-6] employ a series of such unitary processes [7-11] to carry out computations using $d$-dimensional systems as resources. Thus, reliable characterizations of quantum processes are crucial prerequisites for enhancing the quality of quantum technologies. Such a characterization conventionally requires $O\left(d^{4}\right)$ measurements [12-16] that are too resource intensive to perform for large $d$. Ancilla[17-20] and error-correction-based [21-24] quantum process tomography (QPT) schemes were introduced to circumvent this problem. These demand sophisticated state and measurement preparations. For specific property prediction tasks, direct schemes may be sufficient [25-30].

When the unknown process has a certain maximum possible rank, the concept of compressed sensing [31-37] has so far been the status quo for reconstructing the unknown process with a small set of specialized measurements [38-40]. In practice however, this concept is only as reliable as the accuracy of the rank knowledge, and lacks an independent verification method to check the reconstruction results without fidelity comparison with target processes [39,40]. Existing remedies for tackling these issues in compressed sensing are generally ad hoc and incomplete [41].
In what follows, we shall present and experimentally demonstrate an adaptive compressive quantum process tomography scheme (ACQPT) that uniquely characterizes any process through direct diagonal-element-probing measurements in optimally chosen bases that are much fewer than $O\left(d^{4}\right)$, ergo highly compressive. Our scheme does not rely on any sort of prior assumption about the process, with the exception that one knows the dimension $d$ of the underlying quantum system. Instead, it is designed to extract information that is already inherently encoded in the measured data to reveal all process-matrix elements and check if they are uniquely consistent with the data using an efficient semidefinite program $[42,43]$. If not, the scheme adaptively chooses the next optimal measurement to perform, and repeats itself until the process is uniquely characterized.

We shall elaborate the theoretical formalism of ACQPT, and demonstrate that it is both highly compressive and achievable in practice using a proof-of-principle quantum optics experiment for two-qubit processes. Numerical simulations of a range of dimensions supply compelling evidence of an $O\left(d^{2}\right) \ll O\left(d^{4}\right)$ measurement cost for characterizing qudit unitary processes, while experimental data confirm the robustness of ACQPT in the presence of statistical noise.

Compressive characterization of physical processes.Every quantum process that governs natural phenomena can be completely described by a positive semidefinite matrix $\chi$, which is defined by $O\left(d^{4}\right)$ parameters [12]. This matrix represents a state-to-state transformation rule for the 


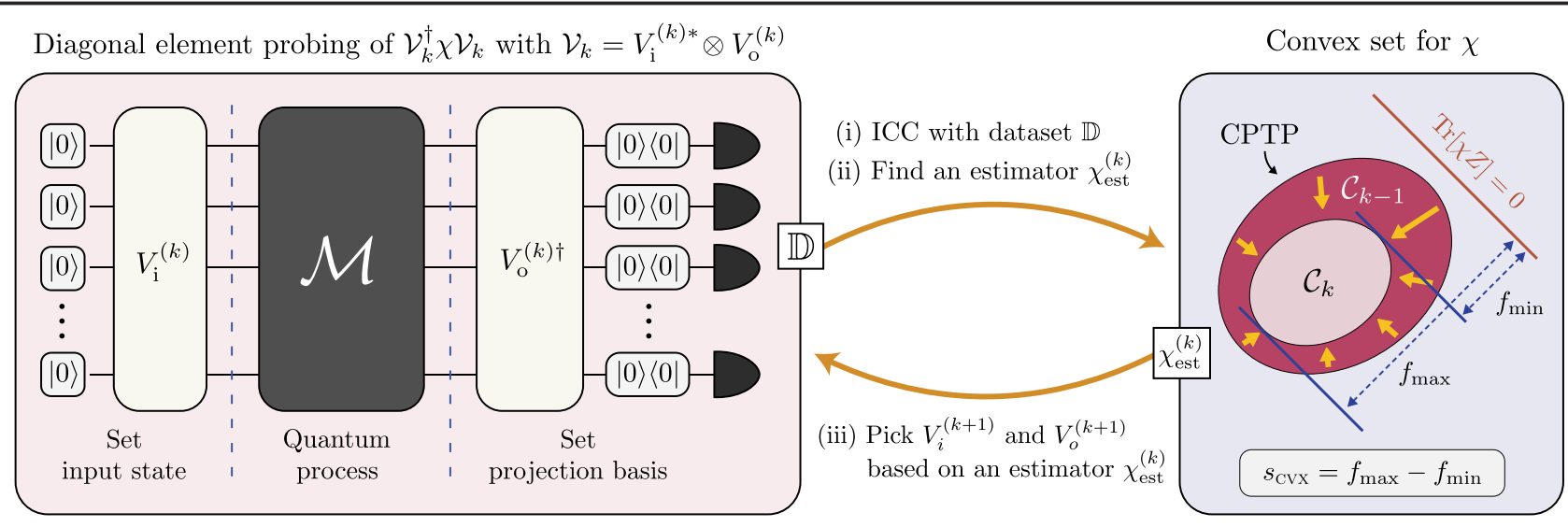

FIG. 1. An iterative schematic of adaptive compressive quantum process tomography (ACQPT) to find out the $\chi$ matrix that represents a quantum process $\mathcal{M}$. This schematic is feasible in practice and generalizes to systems of any dimension, making ACQPT universal. At a particular step $k$, the input state and projection basis are set by the control unitaries $V_{i}^{(k)}$ and $V_{o}^{(k)}$ that defines the $\chi$ rotation of $\mathcal{V}_{k}^{\dagger} \chi \mathcal{V}_{k}$ with $\mathcal{V}_{k}=V_{i}^{(k) *} \otimes V_{o}^{(k)}$. The detection probability that corresponds to the first diagonal element is accumulated in the dataset $\mathbb{D}$. Because the diagonal element probing of $U_{k}^{\dagger} \chi U_{k}$ in general demands resource-intensive measurements, $U_{k}^{\dagger} \chi U_{k}$ is replaced by the closely approximated $\mathcal{V}_{k}^{\dagger} \chi \mathcal{V}_{k}$ whose diagonal element can be measured with the simple experimental setup. Completely positive and tracepreserving process (CPTP) matrices $\chi$ that satisfy the dataset $\mathbb{D}$ form a convex $\operatorname{set} \mathcal{C}_{k}$, which shrinks as data accumulate. In informational completeness certification (ICC), we track this shrinkage with a linear function $f(\chi)=\operatorname{Tr}[\chi Z] / \sqrt{\operatorname{Tr}\left[Z^{2}\right]}$, where $Z$ is a full-rank positive operator. Accordingly, the size monotone $s_{\mathrm{CVX}}$ is defined as the difference between its unique maximum $f_{\max }$ and minimum $f_{\min }$ over $\mathcal{C}_{k}$. If $\mathcal{C}_{k}$ contains only a single estimator $\left(s_{\mathrm{CVX}}=0\right)$, then this unique estimator $\chi_{\text {est }}^{(k)}$ is used to represent the quantum process and terminate ACQPT. If not, ACQPT picks an optimal estimator $\chi_{\text {est }}^{(k)}$ having the minimum entropy from the convex set $\mathcal{C}_{k}$ to pick the next $V_{i}^{(k+1)}$ and $V_{o}^{(k+1)}$.

process that accounts for all physical characteristics of the quantum system. We shall unambiguously determine $\chi$ using the minimal number of measurements necessary with no other presumed information apart from knowing the dimension $d$ of the system $\left(\operatorname{dim}\{\chi\}=d^{2}\right)$. Without loss of generality, we shall investigate trace-preserving processes, examples of which include all unitary processes used in quantum computation. All subsequent discussions directly apply also to non-trace-preserving processes if so desired.

Characterizing a physical process is equivalent to unambiguously finding out the elements of $\chi$. The aim is to do this with as little measurement resources as possible without the need for any other a priori information about $\chi$. We first emphasize a pivotal observation about rankdeficient $\chi$ matrices: When the unknown process is unitary, its $\chi$ matrix is rank 1 and possesses only one positive eigenvalue. Then, we just need to diagonalize $\chi$ via its diagonalizing unitary matrix $U_{\text {diag }}$ and measure that single eigenvalue to fully characterize $\chi$ (The trace-preserving condition would have fixed this eigenvalue anyway so no measurement is even needed in this case). This straightforward argument can be extended to a rank- $r$ process. In this case, we simply measure all $r-1$ positive diagonal values of $U_{\text {diag }}^{\dagger} \chi U_{\text {diag. }}$. In other words, diagonal-element measurements, in view of the prior knowledge about $U_{\text {diag }}$, supply the most information compared to other kinds of measurements.
Evidently, as one has no knowledge about $\chi, U_{\text {diag }}$ is also unknown. Nonetheless, we can design ACQPT to adaptively choose a sequence of unitary rotations $\mathcal{U}=$ $\left\{U_{1}, U_{2}, \ldots, U_{k}, \ldots\right\}$ on $\chi$ that converges to $U_{\text {diag. }}$. Put simply, the iterative scheme executes two basic stages per step. In stage (i), the scheme deterministically certifies if the accumulated dataset $\mathbb{D}$ from the experiment corresponds to a unique estimator $\chi_{\text {est }}$ for the unknown $\chi$ matrix-the informationally complete (IC) situation. The contrary would imply that there is a convex set of processes $\mathcal{C}$ consistent with the non-IC $\mathbb{D}[44,45]$. We call this the informational completeness certification (ICC) stage, and its successful implementation stems from the convexity property of $\mathcal{C}$ that allows us to assign a mathematically justified number $s_{\mathrm{CVX}}$ (size monotone) to indicate whether $\mathbb{D}$ is IC $\left(s_{\mathrm{CVX}}=0\right)$ or not.

For a realistic numerical approach, we preset a certain threshold $\varepsilon$ of $s_{\mathrm{CVX}}$. If $s_{\mathrm{CVX}}>\varepsilon$, ACQPT finds an optimal measurement setting to collect more data in stage (ii) based on $\mathbb{D}$ alone. This is the adaptive measurement stage responsible for generating $\mathcal{U}$. Since our $\chi$ of interest is rank deficient, we can turn ACQPT into a compressive scheme by employing an effective low-rank guiding prescription: After ICC, an estimator with the minimum von Neumann entropy (minENT) is chosen from $\mathcal{C}$ [46-49]. The next optimal $U$ for the $\chi$ rotation would be the one that diagonalizes this estimator to be the eigenvalues in descending order. 


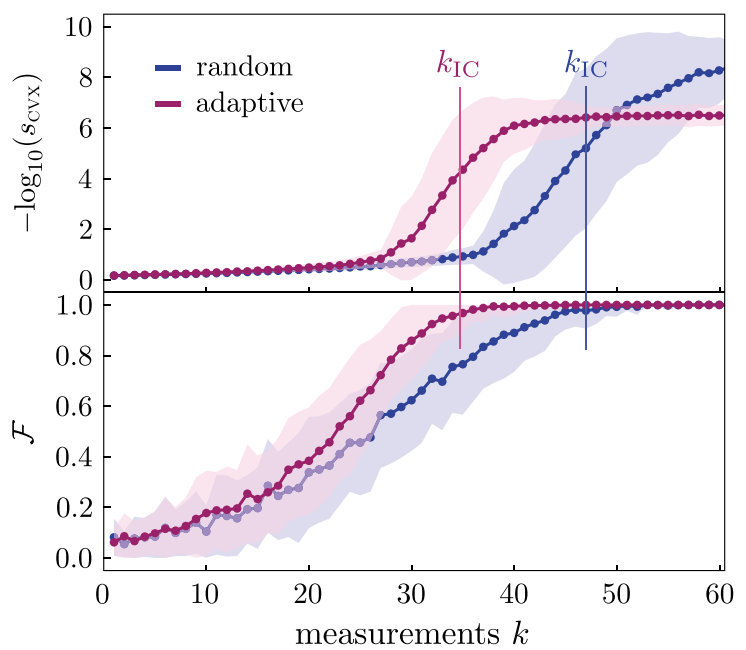

FIG. 2. Numerical simulations of adaptive and random strategies for $s_{\mathrm{CVX}}$ and fidelity $\mathcal{F}$ with ACQPT for minimal nontrivial ququart unitary processes. A random rotation sequence is defined by Haar-random unitary matrices (see Supplemental Material, Sec. II [50]) and $\mathcal{F}$ in the $k$ th iterative step is computed using the optimal $\chi_{\text {est }}^{(k)}$ chosen with minENT. The markers and shaded regions indicate the average values and standard deviations respectively over 60 random ququart unitary processes. Here, the $k_{\text {IC }}$ values are recorded at the instant $s_{\mathrm{CVX}}<5 \times 10^{-5}$, which are respectively $34.7 \pm 3.6$ and $47.0 \pm 5.9$ for the adaptive and random measurements. All simulations are statistically noiseless, so that $\mathcal{F}$ at $k_{\mathrm{IC}}$ for every process is unity to numerical precision.

At the $k$ th iterative step, the $\kappa_{k}$ th diagonal element of $U_{k}^{\dagger} \chi U_{k}$ is measured following the rule $\kappa_{k}=\bmod \left(k, r_{k-1}\right)+1$, where $r_{k-1}=\operatorname{rank}\left\{\chi_{\text {est }}^{(k-1)}\right\}$. The logic of this "modulo rule" is to measure the diagonal element of the cyclically shifted index within the positive-eigenvalue sector of the previously estimated $\chi_{\text {est }}$, such that eventually all positive eigenvalues of $\chi$ are measured (up to statistical noise) at step $k=k_{\mathrm{IC}}$, that is the final step at which IC measurement data are obtained. As an example, ACQPT measures the first diagonal element of $\chi$ at $k=1$, then the second diagonal element of $U_{2}^{\dagger} \chi U_{2}$ at $k=2$, and back to the first diagonal element of $U_{3}^{\dagger} \chi U_{3}$ if $r_{2}=\operatorname{rank}\left\{\chi_{\text {est }}^{(2)}\right\}<3$, and so forth.

Figure 1 shows an iterative schematic of ACQPT. The diagonal element probing of $U_{k}^{\dagger} \chi U_{k}$ in general demands resource-intensive measurements, thus we approximate the probing in an experimentally feasible way using a variable input $V_{i}^{(k)}|0 \ldots 0\rangle$ and projection onto $V_{o}^{(k)}|0 \ldots 0\rangle$. The first diagonal element of $\mathcal{V}_{k}^{\dagger} \chi \mathcal{V}_{k}$, where $\mathcal{V}_{k}=V_{i}^{(k) *} \otimes V_{o}^{(k)}$, can be obtained from the detection probability, and $V_{i}^{(k)}$ and $V_{o}^{(k)}$ are chosen to closely approximate the $\kappa_{k}$ th diagonal of $U_{k}^{\dagger} \chi U_{k}$. We invite the reader to visit the Supplemental Material, Sec. I [50], for further elaboration.

As ACQPT proceeds, more independent data are collected such that $U_{k} \rightarrow U_{\text {diag }}$ quickly. In this way, our scheme can efficiently acquire optimal data and determine

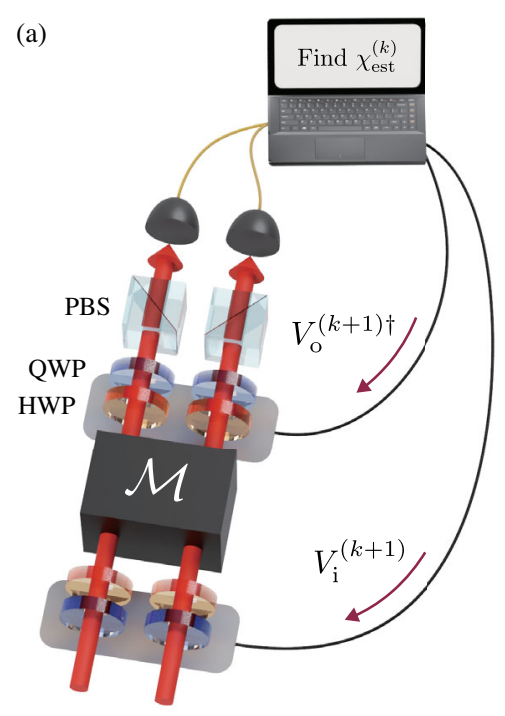

(b) $\mathbb{I} \otimes H$

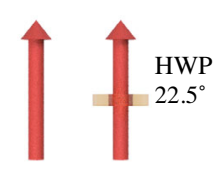

(c) Controlled-NOT

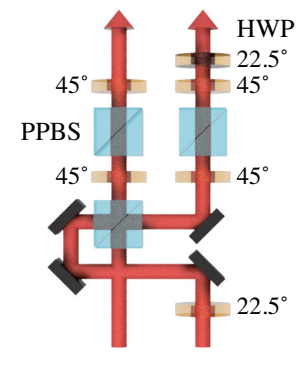

FIG. 3. (a) The polarization state of two photons is transformed by a two-qubit process $\mathcal{M}$. For ACQPT on the unknown $\chi$ matrix that represents $\mathcal{M}$, the initial state and output projection basis are adaptively manipulated by controlling half-wave plates (HWPs) and quarter-wave plates (QWPs) according to $V_{i}^{(k+1)}$ and $V_{o}^{(k+1)}$, which are given from the previously estimated $\chi_{\text {est }}^{(k)}$ based on the accumulated dataset (see Supplemental Material, Sec. I [50]). As the target two-qubit processes, $\mathbb{I} \otimes H$ (Hadamard transform only on the second qubit) and controlled-NOT (CNOT) gates are optically constructed. (b) A HWP at $22.5^{\circ}$ is placed on the second qubit, which functions as the Hadamard gate. (c) Only if two single photons have vertical polarization, the Hong-Ou-Mandel interference on a partially polarizing beam splitter (PPBS) imprints a phase shift of $\pi$ onto two-qubit state, that is, controlled-phase gate. To make a CNOT gate, HWPs at $22.5^{\circ}$ are applied on the second (target) qubit before and after the controlled-phase gate [51,52].

whether they are sufficient to uniquely recover $\chi$ without ever requiring spurious preexperimental assumptions about $\chi$. Notice that the rank deficiency of $\chi$ is not assumed here. A (nearly) full-rank $\chi$ unbeknownst to us would automatically result in a much slower convergence of ACQPT.

Numerical results.-Our first numerical showcase of ACQPT demonstrates the superiority of adaptive $\chi$-rotation sequences over random ones. For this, both the size monotone $s_{\mathrm{CVX}}$ and fidelity $\mathcal{F}$ with the true process are numerically simulated with multiple randomly chosen ququart $(d=4)$ unitary processes (see Fig. 2). The results clearly show that the adaptive strategy gives a significantly smaller $k_{\mathrm{IC}}$ than the random strategy.

We further give numerical estimates on the scaling behaviors of $k_{\mathrm{IC}}$ for both the adaptive and random strategies on qudit unitary processes in the Supplemental Material, Sec. III [50]. We show, for a reasonably large range of $d$ and multiple simulations with random processes, that these strategies only need measurement resources of $O\left(d^{2}\right)$ in contrast with the standard $O\left(d^{4}\right)$. For a more complete analysis, we also compared the minENT strategy in 
(a)
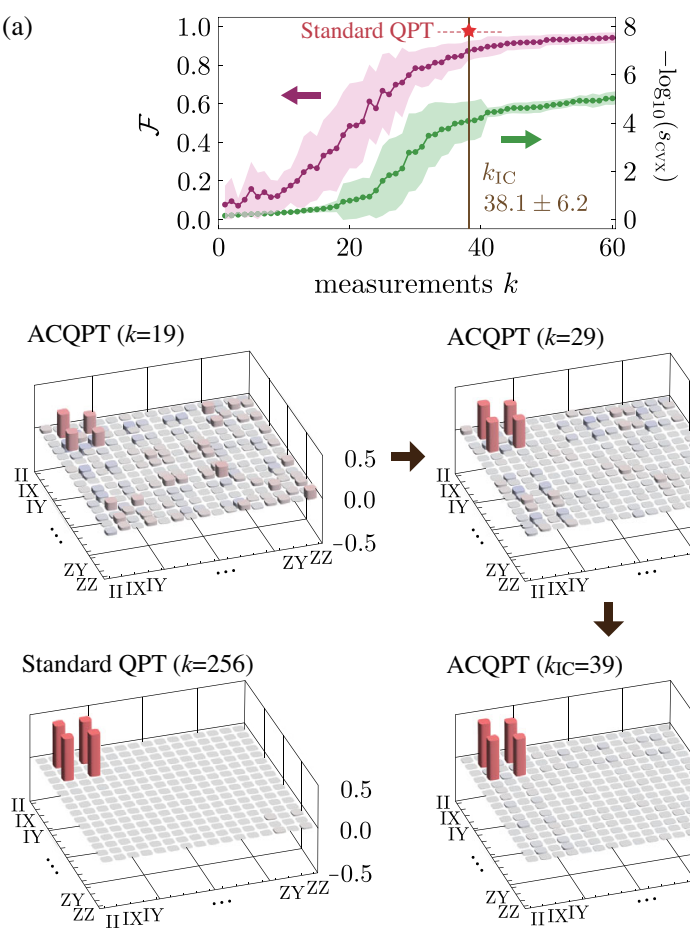

$\operatorname{ACQPT}(k=29)$
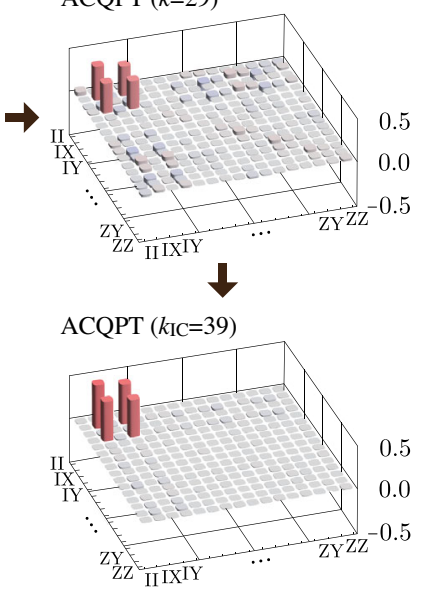

(b)
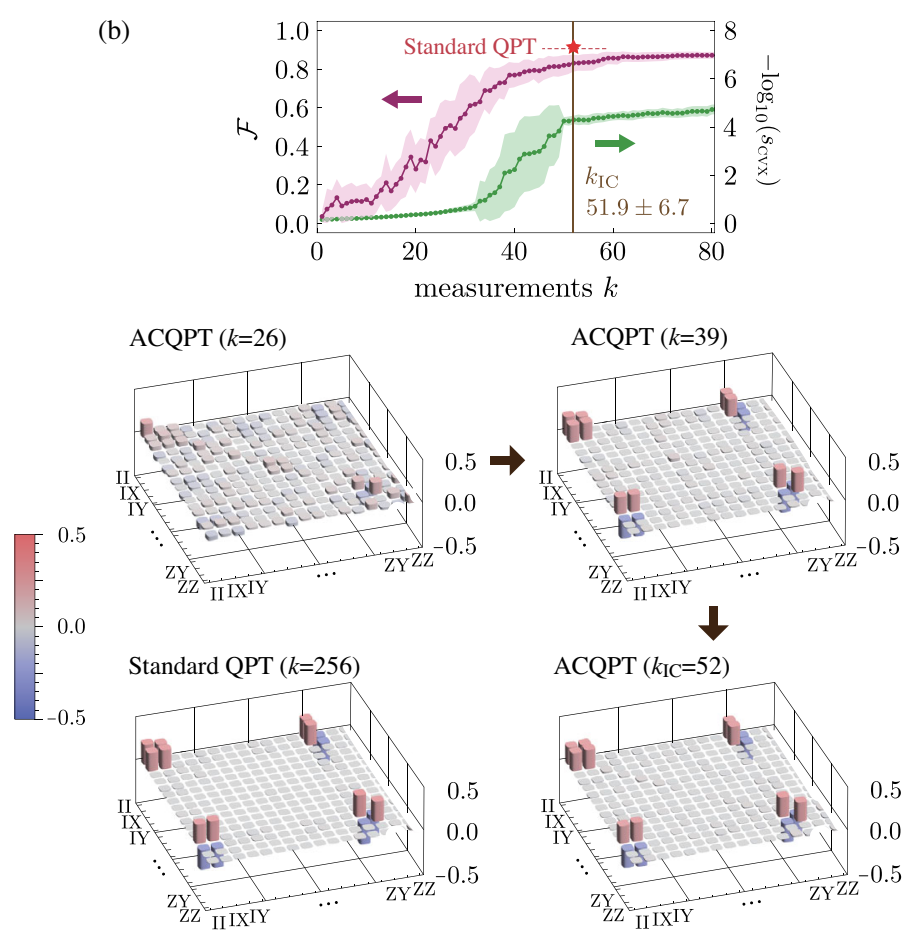

FIG. 4. The experimental ACQPT results plotted for both the (a) $\mathbb{I} \otimes H$ and (b) CNOT gates. All $k_{\mathrm{IC}}$ values are recorded at the instants when $s_{\mathrm{CVX}}$ drops below $5 \times 10^{-5}$, and 15 experimental runs are performed on each gate. Although both gates should ideally be unitary, the CNOT gate possesses a $\chi$ matrix of higher rank due to the hardware imperfections such as polarization-dependent losses and partial distinguishability within a photon pair. Accordingly, the CNOT gate requires more measurements than the $\mathbb{I} \otimes H$ gate, and hence a larger $k_{\mathrm{IC}}$ and a lower IC $\mathcal{F}$. The density-matrix plots of the minENT estimator $\chi_{\text {est }}^{(k)}$ at various ACQPT step numbers $k$ for each gate paint the evolution picture of the compression run that leads to the final unique estimator $\chi_{\text {est }}^{\left(k_{\mathrm{IC}}\right)}$, which is close to the standard QPT reconstruction using $k=256$ measurements.

ACQPT with another available adaptive strategy, the minimum-L1 norm strategy.

Experimental results.-The experimental platform we use for demonstrating ACQPT utilizes a source of two photons. The quantum processes of interest are implemented for the polarization modes. A 140-fs ultrafast laser is impinged on a $1 \mathrm{~mm}$-thick type-II Barium Borate (BBO) crystal to emit two single photons in the beamlike configuration using spontaneous parametric down conversion (SPDC). The individual photons delivered to the experimental setup shown in Fig. 3(a) with single-mode fibers each possesses the central wavelength of $780 \mathrm{~nm}$. To maximize the indistinguishability between two photons, their spectra are truncated by making use of interference filters having $2 \mathrm{~nm}$ full-width at half-maximum bandwidth.

The two-photon state is first initialized to the doubly horizontally polarized state $|00\rangle\langle 00|$. At the $k$ th iterative step of ACQPT, the initial state and projection basis are manipulated using half- (HWP) and quarter-wave (QWP) plates according to the previously chosen two-qubit operations $V_{i}^{(k)}$ and $V_{o}^{(k)}$, respectively. For simple implementation, the closest separable initial state and projection basis are utilized. We anticipate an even better performance from ACQPT with sophisticated entangling operations.
The ACQPT scheme proceeds as follows: Starting with $k=1, V_{i}^{(1)}$ and $V_{o}^{(1)}$ are generated randomly because there is initially no information about the unknown $\chi$. Making use of the algorithm in the Supplemental Material, Sec. I [50], a datum $\mathbb{D}_{1}$ is obtained from the probability of coincidentally detecting a photon at each of the two photodetectors [see Fig. 3(a)]. This probability is estimated from dividing the coincidence counts for the setting of $V_{i}^{(1)}$ and $V_{o}^{(1)}$ by the total input photon counts, the latter of which can be measured by removing the polarizing beam splitter (PBS) at once. The datum is then utilized to find $V_{i}^{(2)}$ and $V_{o}^{(2)}$ to measure in the next iteration, and the whole procedure repeats until a unique process matrix is completely characterized at $k=k_{\mathrm{IC}}$.

We investigate both the $\mathbb{I} \otimes H$ (Hadamard transform only on the second qubit) and controlled-NOT (CNOT) gates constructed as in Figs. 3(b) and 3(c). The Hadamard gate is simply realized using a HWP at $22.5^{\circ}$, whereas the CNOT gate is implemented by exploiting Hong-OuMandel interference effects on a partially polarizing beam splitter (PPBS) that partially reflects vertical polarization with a transmittance of $1 / 3$ and perfectly transmits horizontal polarization [51-53]. Figure 4 shows plots of $s_{\mathrm{CVX}}$ 
and $\mathcal{F}$ at each step $k$ for the two-qubit processes. ACQPT essentially gives almost the same results as standard QPT with much fewer measurement outcomes $(38.1 \pm 6.2$ and $51.9 \pm 6.7$ for $\mathbb{I} \otimes H$ and CNOT gates) than 256 and no prior information. The disparity in the required number of measurements for the two gates stems from their different degrees of implementation imperfections that result in nonunitary processes. This clearly shows that ACQPT works adaptively.

Discussion.-All presented simulation and experimental results have confirmed, indeed, that our adaptive elementprobing compressive scheme can characterize any quantum process using drastically less measurement resources than the standard $O\left(d^{4}\right)$ without imposing $a d$ hoc assumptions. Additional simulation graphs and procedures illustrated in the Supplemental Material, Sec. III [50], provide evidence that for general qudit unitary processes, there exists a quadratic enhancement to $O\left(d^{2}\right)$ in terms of measurement resource costs needed to unambiguously characterize any qudit unitary process in contrast with $O\left(d^{4}\right)$.

One may additionally incorporate trusted prior information into ACQPT. Most straightforwardly, if one insists in knowing the rank $r$ of the actual unknown process, then one may simply replace $r_{k}$ with $r$ in the "modulo rule" when measuring diagonal elements. Numerically for example, if we enforce $r=1$, ACQPT becomes comparable to the most efficient Baldwin-Kalev-Deutsch (BKD) scheme [38,54] for a unitary channel known to date, that requires projective measurements of $2 d^{2}-d$. See the Supplemental Material, Sec. III [50], for details. The advantage of incorporating the prior information this way into ACQPT is that even if the prior information turns out to be inaccurate, the effect is not detrimental since an additional layer of certification is carried out to verify if the process estimator is truly unique. This failsafe is what distinguishes ACQPT in merit from all other reported undersampled characterization schemes to the authors' knowledge.

Furthermore, as shown in Fig. 2, the size monotone and fidelity progress very similarly for both the adaptive and random strategies during the initial measurement phase. We may then use this observation to arrive at a hybrid compressive scheme where random measurements are first used at the initial phase before they are switched to adaptive ones. This would also further reduce the overall computational load in trying to execute the adaptive stage repeatedly.

This work was supported in part by the National Research Foundation of Korea (NRF) (Grants No. 2019R1A2C3004812, No. 2019M3E4A1080074, No. 2019R1H1A3079890, No. 2020R1A2C1008609, and No. 2019R1A6A1A10073437), KIST institutional program (Project No. 2E30620), and the Spanish MINECO (Grants No. FIS201567963-P and No. PGC2018-099183-B-I00). Y. K. acknowledges support from the Global Ph.D. Fellowship by the NRF (Grant No. 2015H1A2A1033028).

Y. K. and Y. S. T. contributed equally to this work.

*ys_teo@snu.ac.kr

jeongh@snu.ac.kr

yoonho72@gmail.com

[1] Y.-C. Jeong, J.-C. Lee, and Y.-H. Kim, Experimental implementation of a fully controllable depolarizing quantum operation, Phys. Rev. A 87, 014301 (2013).

[2] H.-T. Lim, K.-H. Hong, and Y.-H. Kim, Experimental demonstration of high fidelity entanglement distribution over decoherence channels via qubit transduction, Sci. Rep. 5, 15384 (2015).

[3] Y.-S. Kim, J.-C. Lee, O. Kwon, and Y.-H. Kim, Protecting entanglement from decoherence using weak measurement and quantum measurement reversal, Nat. Phys. 8, 117 (2012).

[4] T. D. Ladd, F. Jelezko, R. Laflamme, Y. Nakamura, C. Monroe, and J. L. O'Brien, Quantum computers, Nature (London) 464, 45 (2010).

[5] E. T. Campbell, B. M. Terhal, and C. Vuillot, Roads towards fault-tolerant universal quantum computation, Nature (London) 549, 172 (2017).

[6] B. Lekitsch, S. Weidt, A. G. Fowler, K. Mølmer, S. J. Devitt, C. Wunderlich, and W. K. Hensinger, Blueprint for a microwave trapped ion quantum computer, Sci. Adv. 3, e1601540 (2017).

[7] V. M. Schäfer, C. J. Ballance, K. Thirumalai, L. J. Stephenson, T. G. Ballance, A. M. Steane, and D. M. Lucas, Fast quantum logic gates with trapped-ion qubits, Nature (London) 555, 75 (2018).

[8] X.-F. Shi, Accurate Quantum Logic Gates by Spin Echo in Rydberg Atoms, Phys. Rev. Applied 10, 034006 (2018).

[9] T. Ono, R. Okamoto, M. Tanida, H. F. Hofmann, and S. Takeuchi, Implementation of a quantum controlled-swap gate with photonic circuits, Sci. Rep. 7, 45353 (2017).

[10] R. B. Patel, J. Ho, F. Ferreyrol, T. C. Ralph, and G. J. Pryde, A quantum Fredkin gate, Sci. Adv. 2, e1501531 (2016).

[11] J. Fiurášek, Linear optical Fredkin gate based on partialswap gate, Phys. Rev. A 78, 032317 (2008).

[12] I. Chuang and M. Nielsen, Quantum Computation and Quantum Information (Cambridge University Press, Cambridge, England, 2000).

[13] J. L. O’Brien, G. J. Pryde, A. Gilchrist, D. F. V. James, N. K. Langford, T. C. Ralph, and A. G. White, Quantum Process Tomography of a Controlled-Not Gate, Phys. Rev. Lett. 93, 080502 (2004).

[14] J. Fiurášek, Maximum-likelihood estimation of quantum measurement, Phys. Rev. A 64, 024102 (2001).

[15] J. F. Poyatos, J. I. Cirac, and P. Zoller, Complete Characterization of a Quantum Process: The Two-Bit Quantum Gate, Phys. Rev. Lett. 78, 390 (1997).

[16] Y.S. Teo, B.-G. Englert, J. Řeháček, and Z. Hradil, Adaptive schemes for incomplete quantum process tomography, Phys. Rev. A 84, 062125 (2011).

[17] J. B. Altepeter, D. Branning, E. Jeffrey, T. C. Wei, P. G. Kwiat, R. T. Thew, J. L. O'Brien, M. A. Nielsen, 
and A. G. White, Ancilla-Assisted Quantum Process Tomography, Phys. Rev. Lett. 90, 193601 (2003).

[18] D. W. Leung, Choi's proof as a recipe for quantum process tomography, J. Math. Phys. (N.Y.) 44, 528 (2003).

[19] G. M. D'Ariano and P. Lo Presti, Imprinting Complete Information About a Quantum Channel on its Output State, Phys. Rev. Lett. 91, 047902 (2003).

[20] G. M. D'Ariano and P. Lo Presti, Quantum Tomography for Measuring Experimentally the Matrix Elements of an Arbitrary Quantum Operation, Phys. Rev. Lett. 86, 4195 (2001).

[21] S. Omkar, R. Srikanth, and S. Banerjee, Characterization of quantum dynamics using quantum error correction, Phys. Rev. A 91, 012324 (2015).

[22] S. Omkar, R. Srikanth, and S. Banerjee, Quantum code for quantum error characterization, Phys. Rev. A 91, 052309 (2015).

[23] M. Mohseni and D. A. Lidar, Direct characterization of quantum dynamics: General theory, Phys. Rev. A 75, 062331 (2007).

[24] M. Mohseni and D. A. Lidar, Direct Characterization of Quantum Dynamics, Phys. Rev. Lett. 97, 170501 (2006).

[25] Y. Kim, Y.-S. Kim, S.-Y. Lee, S.-W. Han, S. Moon, Y.-H. Kim, and Y.-W. Cho, Direct quantum process tomography via measuring sequential weak values of incompatible observables, Nat. Commun. 9, 192 (2018).

[26] A. Gaikwad, D. Rehal, A. Singh, Arvind, and K. Dorai, Experimental demonstration of selective quantum process tomography on an nmr quantum information processor, Phys. Rev. A 97, 022311 (2018).

[27] A. Bendersky and J. P. Paz, Selective and efficient quantum state tomography and its application to quantum process tomography, Phys. Rev. A 87, 012122 (2013).

[28] C. T. Schmiegelow, A. Bendersky, M. A. Larotonda, and J. P. Paz, Selective and Efficient Quantum Process Tomography Without Ancilla, Phys. Rev. Lett. 107, 100502 (2011).

[29] A. Bendersky, F. Pastawski, and J. P. Paz, Selective and efficient quantum process tomography, Phys. Rev. A 80, 032116 (2009).

[30] A. Bendersky, F. Pastawski, and J. P. Paz, Selective and Efficient Estimation of Parameters for Quantum Process Tomography, Phys. Rev. Lett. 100, 190403 (2008).

[31] D. Donoho, Compressed sensing, IEEE Trans. Inf. Theory 52, 1289 (2006).

[32] E. J. Candés and T. Tao, Near-optimal signal recovery from random projections: Universal encoding strategies?, IEEE Trans. Inf. Theory 52, 5406 (2006).

[33] E. J. Candés and B. Recht, Exact matrix completion via convex optimization, Found. Comput. Math. 9, 717 (2009).

[34] D. Gross, Y.-K. Liu, S. T. Flammia, S. Becker, and J. Eisert, Quantum State Tomography Via Compressed Sensing, Phys. Rev. Lett. 105, 150401 (2010).

[35] A. Kalev, R. L. Kosut, and I. H. Deutsch, Quantum tomography protocols with positivity are compressed sensing protocols, npj Quantum Inf. 1, 15018 (2015).

[36] A. Steffens, C. A. Riofrío, W. McCutcheon, I. Roth, B. A. Bell, A. McMillan, M. S. Tame, J. G. Rarity, and J. Eisert,
Experimentally exploring compressed sensing quantum tomography, Quantum Sci. Technol. 2, 025005 (2017).

[37] C. A. Riofrío, D. Gross, S. T. Flammia, T. Monz, D. Nigg, R. Blatt, and J. Eisert, Experimental quantum compressed sensing for a seven-qubit system, Nat. Commun. 8, 15305 (2017).

[38] C. H. Baldwin, A. Kalev, and I. H. Deutsch, Quantum process tomography of unitary and near-unitary maps, Phys. Rev. A 90, 012110 (2014).

[39] A. V. Rodionov, A. Veitia, R. Barends, J. Kelly, D. Sank, J. Wenner, J. M. Martinis, R. L. Kosut, and A. N. Korotkov, Compressed sensing quantum process tomography for superconducting quantum gates, Phys. Rev. B 90, 144504 (2014).

[40] A. Shabani, R. L. Kosut, M. Mohseni, H. Rabitz, M. A. Broome, M. P. Almeida, A. Fedrizzi, and A. G. White, Efficient Measurement of Quantum Dynamics Via Compressive Sensing, Phys. Rev. Lett. 106, 100401 (2011).

[41] A. Shchukina, P. Kasprzak, R. Dass, M. Nowakowski, and K. Kazimierczuk, Pitfalls in compressed sensing reconstruction and how to avoid them, J. Biomol. NMR 68, 79 (2017).

[42] S. Boyd and L. Vandenberghe, Convex Optimization (Cambridge University Press, Cambridge, England, 2004).

[43] L. Vandenberghe and S. Boyd, Semidefinite programming, SIAM Rev. 38, 49 (1996).

[44] R. Kosut, I. A. Walmsley, and H. Rabitz, Optimal experiment design for quantum state and process tomography and hamiltonian parameter estimation, arXiv:quant-ph/0411093.

[45] R. Kosut, Quantum process tomography via L1-norm minimization, arXiv:0812.4323.

[46] D. Ahn, Y. S. Teo, H. Jeong, F. Bouchard, F. Hufnagel, E. Karimi, D. Koutný, J. Řeháček, Z. Hradil, G. Leuchs, and L. L. Sánchez-Soto, Adaptive Compressive Tomography With No a Priori Information, Phys. Rev. Lett. 122, 100404 (2019).

[47] D. Ahn, Y.S. Teo, H. Jeong, D. Koutný, J. Řeháček, Z. Hradil, G. Leuchs, and L. L. Sánchez-Soto, Adaptive compressive tomography: A numerical study, Phys. Rev. A 100, 012346 (2019).

[48] S. Huang, D. N. Tran, and T. D. Tran, Sparse signal recovery based on nonconvex entropy minimization, in IEEE Trans. Signal Process. 67, 1322 (2019).

[49] D. N. Tran, S. Huang, S. P. Chin, and T. D. Tran, Low-rank matrices recovery via entropy function, in 2016 IEEE International Conference on Acoustics, Speech and Signal Processing (ICASSP) (IEEE, 2016), p. 4064-4068, https:// dx.doi.org/10.1109/ICASSP.2016.7472441.

[50] See Supplemental Material at http://link.aps.org/ supplemental/10.1103/PhysRevLett.124.210401 contains the novel theory of adaptive compressive quantum process tomography that explicitly describes its formalism, practical implementation, a way to cope with statistical noise, and its precise numerical procedure. It also includes miscellaneous recipes for generating random matrices, and additional simulation graphs that illustrate more properties concerning our universal compressive scheme, which was also highlighted in the Letter. 
[51] N. Kiesel, C. Schmid, U. Weber, R. Ursin, and H. Weinfurter, Linear Optics Controlled-Phase Gate Made Simple, Phys. Rev. Lett. 95, 210505 (2005).

[52] R. Okamoto, H. F. Hofmann, S. Takeuchi, and K. Sasaki, Demonstration of an Optical Quantum Controlled-NOT Gate without Path Interference, Phys. Rev. Lett. 95, 210506 (2005).
[53] C. K. Hong, Z. Y. Ou, and L. Mandel, Measurement of Subpicosecond Time Intervals Between Two Photons by Interference, Phys. Rev. Lett. 59, 2044 (1987).

[54] J. Finkelstein, Pure-state informationally complete and really complete measurements, Phys. Rev. A 70, 052107 (2004). 